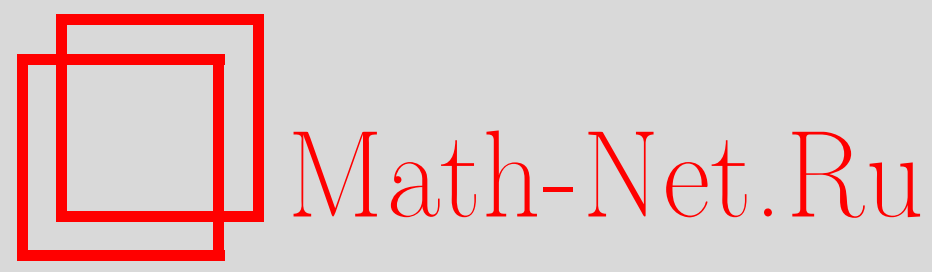

В. Л. Крепс, О теоретико-игровой характеризации стохастической независимости, Дискрет. матем., 2010, том 22, выпуск 1, 115-125

DOI: https://doi.org/10.4213/dm1088

Использование Общероссийского математического портала Math-Net.Ru подразумевает, что вы прочитали и согласны с пользовательским соглашением http://www . mathnet.ru/rus/agreement

Параметры загрузки:

IP : 54.209 .52 .79

26 апреля 2023 г., 14:53:40 
УДК 519.833

\title{
О теоретико-игровой характеризации стохастической независимости
}

\author{
() 2010 г. $\quad$ В. Л. Крепс
}

\begin{abstract}
Вводится понятие типа зависимости выборов игроков в конечных бескоалиционных играх. Доказывается, что стохастическая независимость представляет собой единственный тип зависимости, при котором любая конечная бескоалиционная игра имеет ситуацию равновесия по Нэшу в смешанных стратегиях.
\end{abstract}

\section{1. Введение}

В конечных бескоалиционных играх игроки одновременно и без обмена информации выбирают действия (чистые стратегии) из заданных конечных множеств. В результате совместного выбора всеми игроками складывается ситуация. Каждой ситуации в чистых стратегиях соответствует набор выигрышей игроков. Стратегические возможности игрока могут быть расширены введением смешанных стратегий, то есть вероятностных распределений на множестве чистых стратегий.

Традиционно, в теории бескоалиционных игр, начиная с основополагающей работы Дж. фон Неймана [1] для матричных игр и работы Дж. Нэша [2] для конечных бескоалиционных игр нескольких участников, смешанные стратегии игроков, трактуемые как случайные испытания с исходами из множества чистых стратегий, предполагаются стохастически независимыми. Соответственно, выигрыши игроков определяются как математические ожидания выигрышей в чистых стратегиях по мере, равной произведению независимых мер - смешанных стратегий игроков.

Ситуация называется равновесной по Нэшу [3], если ни один игрок не увеличивает свой выигрыш, отклонившись от выбранной стратегии, в то время как другие игроки не изменили свои стратегии. Теорема Нэша [3] устанавливает, что любая конечная бескоалиционная игра имеет ситуацию равновесия в традиционном смешанном расширении.

Предположение о стохастической независимости рандомизированных выборов игроков базируется прежде всего на физической независимости выборов. В книге М. Каца [4] отмечается, что, вообще говоря, стохастическая независимость испытаний не вытекает из их физической независимости. Аналогичное рассуждение см. в статье Н. Н. Воробьева [5].

Рассмотрим пример, в котором два игрока используют для реализации выбранных ими смешанных стратегий один и тот же датчик случайных чисел. У каждого игрока имеется два возможных действия: $a$ и $b$ - действия игрока $1, c$ и $d-$ действия игрока 2. Игроки выбирают свои действия в соответствии со смешанными стратегиями $\left(x_{a}, x_{b}\right), x_{b}=1-x_{a}$, и $\left(y_{c}, y_{d}\right), y_{d}=1-y_{c}$. Датчик выдает случайное число $z$, равномерно распределенное на отрезке $[0,1]$. Игрок 1 выбирает действие $a$, если $z<x_{a}$. Игрок 2 выбирает действие $c$, если $z<y_{c}$. Тогда при условии $x_{a} \leqslant y_{c}$ вероятность $\mathbf{P}(a \cap c)=x_{a}$, вероятность 
$\mathbf{P}(a \cap d)=0$, вероятность $\mathbf{P}(b \cap c)=y_{c}-x_{a}$ и вероятность $\mathbf{P}(b \cap d)=y_{d}$. Заметим, что в силу маргинальных условий $\mathbf{P}(a \cap c)+\mathbf{P}(a \cap d)=x_{a}, \mathbf{P}(a \cap c)+\mathbf{P}(b \cap c)=y_{c}$ и т.д., достаточно выписать одну из совместных вероятностей.

Приведенное совместное распределение соответствует заданному порядку стратегий. Следующее совместное распределение получается усреднением по всем возможным порядкам. Если $x_{a} \leqslant \min \left(y_{c}, y_{d}\right)$, то вероятность $\mathbf{P}(a \cap c)=x_{a} / 2$, вероятность $\mathbf{P}(a \cap d)=x_{a} / 2$, вероятность $\mathbf{P}(b \cap c)=y_{c}-x_{a} / 2$ и вероятность $\mathbf{P}(b \cap d)=y_{d}-x_{a} / 2$. Если $y_{d} \leqslant \min \left(x_{a} \leqslant x_{b}\right)$, то вероятность $\mathbf{P}(a \cap c)=x_{a}-y_{d} / 2$, вероятность $\mathbf{P}(a \cap d)=y_{d} / 2$, вероятность $\mathbf{P}(b \cap c)=x_{b}-y_{d} / 2$ и вероятность $\mathbf{P}(b \cap d)=y_{d} / 2$.

Отказавшись от априорной стохастической независимости выборов игроков, в [5] Н. Н. Воробьев допустил, что при выборе игроками смешанных стратегий вероятность ситуации в чистых стратегиях есть функция (вообще говоря, не обязательно равная произведению) от вероятностей выборов игроками действий, образующих эту ситуацию. В [5] предполагается, что функция одна и та же для всех ситуаций в чистых стратегиях. Н. Н. Воробьев назвал эту функцию типом зависимости и показал, что если все игроки имеют более двух действий, то эта функция равна произведению, то есть единственный тип зависимости - независимость.

В настоящей работе вводится более общее понятие типа зависимости, как отображения, которое каждой ситуации в смешанных стратегиях (каждому набору вероятностных распределений на множествах действий игроков) сопоставляет совместное вероятностное распределение на множестве ситуаций в чистых стратегиях. При этом определении вероятность ситуации в чистых стратегиях зависит не только от вероятностей действий, составляющих эту ситуацию, а от всей ситуации в смешанных стратегиях. Естественно предполагается, что маргинальные распределения совпадают со смешанными стратегиями игроков. Таким образом, отклонение от стохастической независимости становится возможным, если по крайней мере два игрока рандомизируют свои действия.

Доказывается, что стохастическая независимость представляет собой единственный тип зависимости, при котором любая конечная бескоалиционная игра имеет ситуацию равновесия по Нэшу в смешанных стратегиях. Для случая двух игроков этот результат был установлен в работе автора [6].

\section{2. Определения и свойства}

Рассматриваются конечные бескоалиционные игры $N$ лиц размера $m_{1} \times m_{2} \times \ldots \times m_{N}$. Конечная бескоалиционная игра (см. [7] и [8]) задается тройкой

$$
\Gamma=\left\langle I, S_{i}, A_{i}, i \in I\right\rangle,
$$

где $I=\{1, \ldots, N\}-$ множество игроков, $S_{i}=\left\{1, \ldots, m_{i}\right\}-$ множество чистых стратегий игрока $i$, многомерная вещественная матрица $A_{i}=\left[a_{j_{1} \ldots j_{l} \ldots j_{N}}^{i}\right]_{1 \leqslant j_{l} \leqslant m_{l}}$ задает выигрыши игрока $i$ в ситуации $\left(j_{1}, \ldots, j_{l}, \ldots, j_{N}\right)$ (при выборе игроком $l$ стратегии $\left.j_{l}, l=1, \ldots, N\right)$.

Множество смешанных стратегий (вероятностных смесей чистых стратегий) $X_{i}$ игрока $i$ представляет собой симплекс размерности $m_{i}-1$,

$$
X_{i}=\left\{\mathbf{x}_{i}=\left(x_{i 1}, \ldots, x_{i m_{i}}\right), x_{i j} \geqslant 0, \sum_{j=1}^{m_{i}} x_{i j}=1\right\} \text {. }
$$

Чистую стратегию $j$ игрока $i$, как элемент $X_{i}$, будем обозначать $\mathbf{e}_{i}^{j}$. 
При использовании игроками смешанных стратегий $\mathbf{x}_{l}, l=1, \ldots, N$, выигрышу игрока $i, i=1, \ldots, N$, в ситуации $\mathbf{x}=\left(\mathbf{x}_{1}, \ldots, \mathbf{x}_{l}, \ldots, \mathbf{x}_{N}\right)$ предписывается значение

$$
H_{i}(\mathbf{x})=\sum_{j_{1}, \ldots, j_{N}} a_{j_{1} \ldots j_{N}}^{i} \prod_{k=1}^{N} x_{k j_{k}},
$$

что соответствует стохастической независимости выборов игроков.

Стратегия $\mathbf{x}_{i}$ игрока $i$ называется вполне смешанной, если все $x_{i j}>0, j=1, \ldots, m_{i}$. Ситуация $\mathbf{x}=\left(\mathbf{x}_{1}, \ldots, \mathbf{x}_{N}\right)$ называется вполне смешанной, если все входящие в нее стратегии $\mathbf{x}_{i}$ вполне смешаны.

Ситуация $\mathbf{x}^{*}=\left(\mathbf{x}_{1}^{*}, \ldots, \mathbf{x}_{N}^{*}\right)$ называется ситуацией равновесия по Нэшу [3] в игре $\Gamma$, если для всех игроков $i=1, \ldots, N$ справедливы равенства

$$
H_{i}\left(\mathbf{x}^{*}\right)=\max _{\mathbf{x}_{i} \in X_{i}} H_{i}\left(\mathbf{x}^{*} \| \mathbf{x}_{i}\right),
$$

где $\mathbf{x}^{*} \| \mathbf{x}_{i}=\left(\mathbf{x}_{1}^{*}, \ldots, \mathbf{x}_{i-1}^{*}, \mathbf{x}_{i}, \mathbf{x}_{i+1}^{*}, \ldots, \mathbf{x}_{N}^{*}\right)$ - ситуация, в которой все игроки, за исключением игрока $i$, выбрали свои стратегии-компоненты ситуации $\mathbf{x}^{*}$.

Поскольку

$$
x_{l m_{l}}=1-\sum_{j=1}^{m_{l}-1} x_{l j}
$$

функцию выигрыша $H_{i}$ игрока $i$ можно переписать как функцию от $\prod_{k=1}^{N}\left(m_{k}-1\right)$ переменных $x_{l 1}, \ldots, x_{l m_{l}-1}, l=1, \ldots, N$,

$$
H_{i}(\mathbf{x})=\sum_{l=1}^{N} \sum_{j_{1}=l}^{m_{l}-1} b_{j_{1} \ldots j_{N}}^{i} \prod_{k=1}^{N} x_{k j_{k}}+\ldots+\sum_{l=1}^{N} \sum_{j_{l}=1}^{m_{l}-1} b^{i}\left(j_{l}\right) x_{l j_{l}}+b^{i},
$$

где коэффициенты $b_{j_{1} \ldots j_{N}}^{i}, \ldots, b^{i}$ определяются матрицей $A_{i}$.

Легко видеть, что любой $N$-набор функций такого вида соответствует некоторой конечной бескоалиционной игре $N$ лиц размера $m_{1} \times m_{2} \times \ldots \times m_{N}$.

При фиксированных стратегиях других игроков функцию $H_{i}$ можно переписать в виде

$$
H_{i}(\mathbf{x})=\sum_{j=1}^{m_{i}-1} f_{j}^{i}\left(\mathbf{x}^{i}\right) x_{i j}+f^{i}\left(\mathbf{x}^{i}\right),
$$

где $\mathbf{x}^{i}-$ вектор размерности $\prod_{k \neq i}\left(m_{k}-1\right)$, образованный компонентами $x_{k 1}, \ldots, x_{k m_{k}-1}$, $k \neq i$, а $f^{i}$ и $f_{j}^{i}$ - некоторые функции от $\mathbf{x}^{i}$. Отметим, что компоненты $x_{k m_{k}}$ не входят в вектор $\mathbf{x}^{i}$.

Пусть $\mathbf{x}-$ ситуация равновесия в игре Г. Нижеперечисленные свойства 1-3 являются непосредственным следствием определений:

(1) если $f_{j}^{i}\left(\mathbf{x}^{i}\right)<0$ для некоторых $i$ и $j$, то $x_{i j}=0$;

(2) если $f_{j}^{i}\left(\mathbf{x}^{i}\right)>0$ для некоторых $i$ и $j$, то $x_{i m_{i}}=0$;

(3) если ситуация $\mathbf{x}$ вполне смешана, то $f_{j}^{i}\left(\mathbf{x}^{i}\right)=0$ для всех $i=1, \ldots, N$ и $j=1, \ldots, m_{i}-1$. 


\section{3. Отклонение от стохастической независимости}

Допуская возможную зависимость рандомизационных механизмов игроков (например, игроки могут использовать один и тот же генератор случайных чисел), мы отказываемся от априорного предположения о стохастической независимости их выборов.

Отображение $T$, которое каждой ситуации в смешанных стратегиях $\mathbf{x} \in X=\prod_{i=1}^{N} X_{i}$ сопоставляет вероятностное распределение $T(\mathbf{x})$ на множестве ситуаций в чистых стратегиях $S=\prod_{i=1}^{N} S_{i}$, называем $m_{1} \times m_{2} \times \ldots \times m_{N}$-типом зависимости $T$.

Отображение $T: X \rightarrow \Delta(S)$ определяется набором функций

$$
T_{j_{1} \ldots j_{N}}: \quad \prod_{i=1}^{N} X_{i} \rightarrow[0,1], \quad \sum_{j_{1} \ldots j_{N}} T_{j_{1} \ldots j_{N}}\left(\mathbf{x}_{1}, \ldots, \mathbf{x}_{N}\right)=1 .
$$

Мы предполагаем, что для любого фиксированного игрока $k=1, \ldots, N$ и любой его чистой стратегии $j_{k}=1, \ldots, m_{k}$ выполняется равенство

$$
\sum_{i \neq k} \sum_{j_{i}=1}^{m_{i}} T_{j_{1} \ldots j_{k} \ldots j_{N}}\left(\mathbf{x}_{1}, \ldots, \mathbf{x}_{N}\right)=x_{k j_{k}}
$$

то есть одномерные маргинальные распределения совпадают со смешанными стратегиями игроков. Следовательно, если только один игрок рандомизирует свои действия, то не возникает отклонения от стохастической независимости. В случае, если это не вызывает разночтений, мы будем писать тип зависимости $T$, опуская пометку $m_{1} \times m_{2} \times \ldots \times m_{N}$, содержащую число игроков и число их чистых стратегий.

Пример 1. Для случая $m_{i}=2, i=1, \ldots, N$, следующий набор функций дает пример типа зависимости отличного от стохастической независимости. В работе Н. Н. Воробьева [5] этот пример приведен при $N=2$. Пусть

$$
T_{j_{1} \ldots j_{N}}\left(x_{1}, \ldots, x_{N}\right)=\prod_{k=1}^{N} x_{k j_{k}}+\alpha \prod_{k=1}^{N} \sin 2 \pi x_{k j_{k}}
$$

где $j_{k}=1,2,0 \leqslant x_{i} \leqslant 1$, и $\alpha$ достаточно мало.

В смешанном расширении с типом зависимости $T$ положим выигрыш игрока $i$ в ситуации $\mathbf{x}=\left(\mathbf{x}_{1}, \ldots, \mathbf{x}_{N}\right)$ равным математическому ожиданию его выигрышей по мере $T\left(\mathbf{x}_{1}, \ldots, \mathbf{x}_{N}\right)$ :

$$
H_{i}^{T}(\mathbf{x})=\sum_{j_{1}, \ldots, j_{N}} a_{j_{1} \ldots j_{N}}^{i} T_{j_{1} \ldots j_{N}}\left(\mathbf{x}_{1}, \ldots, \mathbf{x}_{N}\right)
$$

Обозначим множество ситуаций равновесия по Нэшу в такой игре $N E(T, \Gamma)$ и, соответственно, в случае стохастической независимости выборов игроков $N E\left(T^{0}, \Gamma\right)$, где

$$
T_{j_{1} \ldots j_{N}}^{0}\left(\mathbf{x}_{1}, \ldots, \mathbf{x}_{N}\right)=\prod_{k=1}^{N} x_{k j_{k}}
$$




\section{4. Маргинальные $(N-1)$-мерные распределения}

Пусть $T-m_{1} \times m_{2} \times \ldots \times m_{N}$-тип зависимости. Фиксируем произвольную смешанную стратегию одного игрока, для определенности стратегию $\mathbf{x}_{1}$ игрока 1. Определим $\left(m_{2}, \ldots, m_{N}\right)$-тип зависимости

$$
T^{\mathbf{x}_{1}}: \quad \prod_{k=2}^{N} X_{k} \rightarrow \Delta\left(\prod_{k=2}^{N} S_{k}\right)
$$

с помощью $(N-1)$-мерных маргинальных распределений на $\prod_{k=2}^{N} S_{k}$ :

$$
T_{j_{2} \ldots j_{N}}^{\mathbf{x}_{1}}\left(\mathbf{x}_{2}, \ldots, \mathbf{x}_{N}\right)=\sum_{j_{1}=1}^{m_{1}} T_{j_{1} j_{2} \ldots j_{N}}\left(\mathbf{x}_{1}, \mathbf{x}_{2}, \ldots, \mathbf{x}_{N}\right) .
$$

Очевидно, что для $T^{\mathbf{x}_{1}}$ маргинальное условие (3) выполняется.

Лемма 1. Если множество $N E(T, \Gamma)$ не пусто для любой конечной бескоалиционной игры $N$ лиц Г размера $m_{1} \times m_{2} \times \ldots \times m_{N}$, то также не пусто множество $N E\left(T^{\mathbf{x}_{1}}, \Gamma^{1}\right)$ для любой игры $(N-1)$ лииа $\Gamma^{1}$ размера $m_{2}, \ldots, m_{N}$.

Доказательство. Игре $(N-1)$ лица $\Gamma^{1}$, задаваемой матрицами $A_{i}^{1}=\left[a_{j_{2} \ldots j_{l} \ldots j_{N}}^{1 i}\right]_{1 \leqslant j_{l} \leqslant m_{l}}$, $i=2, \ldots, N$, сопоставим $m_{1} \times m_{2} \times \ldots \times m_{N}$-игру $N$ лиц $\Gamma$, в которой игрок 1 является “болваном” (как его выигрыш, так и выигрыши остальных игроков не зависят от стратегии игрока 1). Именно, выигрыши игрока 1 тождественно равны нулю, а матрицы выигрышей $A_{i}$ игроков $i=2, \ldots, N$ игры $\Gamma$ не зависят от $j_{1}=1, \ldots, m_{1}$ и задаются равенствами $a_{j_{1} j_{2} \ldots j_{N}}^{i}=a_{j_{2} \ldots j_{N}}^{1 i}$. Легко видеть, что $\left(\mathbf{x}_{2}^{*}, \ldots, \mathbf{x}_{N}^{*}\right)$ является ситуацией равновесия игры $N-1$ лица $\Gamma^{1}$ с типом зависимости $T^{\mathbf{x}_{1}}$, где $\left(\mathbf{x}_{1}^{*}, \mathbf{x}_{2}^{*}, \ldots, \mathbf{x}_{N}^{*}\right)$ - ситуация равновесия игры $\Gamma$ с типом зависимости $T$, которая существует по предположению. Лемма 1 доказана.

Далее в этом разделе мы рассмотрим типы зависимости, для которых при любом $k=1, \ldots, N$ маргинальные $(N-1)$-мерные распределения $T^{\mathbf{x}_{k}}$ не зависят от $\mathbf{x}_{k}, T^{\mathbf{x}_{k}}=T^{k}$ и соответствуют стохастической независимости выборов остальных игроков, то есть выполняются равенства

$$
\sum_{j_{k}=1}^{m_{k}} T_{j_{1} \ldots j_{k} \ldots j_{N}}\left(\mathbf{x}_{1}, \ldots, \mathbf{x}_{N}\right)=\prod_{\substack{i=1 \\ i \neq k}}^{N} x_{i j_{i}}
$$

Из маргинальных условий (4) следует, что отклонение от стохастической независимости не возникает, если хотя бы один игрок не рандомизирует свои действия.

В примере 1 условия (4) выполнены.

Лемма 2. Пусть для $T$ выполняются маргинальные условия (4). Если Г является игрой с нулевой суммой, то $N E(T, \Gamma) \subset N E\left(T^{0}, \Gamma\right)$ и для любой ситуаџии равновесия $\left(\mathbf{x}_{1}^{*}, \ldots, \mathbf{x}_{N}^{*}\right) \in N E(T, \Gamma)$

$$
H_{i}^{T}\left(\mathbf{x}_{1}^{*}, \ldots, \mathbf{x}_{N}^{*}\right)=H_{i}^{T^{0}}\left(\mathbf{x}_{1}^{*}, \ldots, \mathbf{x}_{N}^{*}\right)=\sum_{j_{1} \ldots j_{N}} a_{j_{1} \ldots j_{N}}^{i} \prod_{k=1}^{N} x_{k j_{k}}^{*}, \quad i=1, \ldots, N .
$$


Доказательство. Пусть $\left(\mathbf{x}_{1}^{*}, \ldots, \mathbf{x}_{N}^{*}\right) \in N E(T, \Gamma)$. Учитывая, что если стратегия одного из игроков является чистой, то выполнение маргинальных условий (4) влечет совпадение значений функций $H_{i}^{T}$ и $H_{i}^{T^{0}}$, и принимая во внимание, что максимум функции $H_{i}^{T^{0}}$ достигается на подмножестве чистых стратегий, получаем, что

$$
\begin{aligned}
H_{i}^{T}\left(\mathbf{x}^{*}\right) & =\max _{\mathbf{x}_{i} \in X_{i}} H_{i}^{T}\left(\mathbf{x}_{1}^{*}, \ldots, \mathbf{x}_{N}^{*}\right) \geqslant \max _{j=1, \ldots, m_{i}} H_{i}^{T}\left(\mathbf{x}_{1}^{*}, \ldots, \mathbf{e}_{i}^{j}, \ldots, \mathbf{x}_{N}^{*}\right) \\
& =\max _{j=1, \ldots, m} H_{i}^{T^{0}}\left(\mathbf{x}_{1}^{*}, \ldots, \mathbf{e}_{i}^{j}, \ldots, \mathbf{x}_{N}^{*}\right) \geqslant H_{i}^{T^{0}}\left(\mathbf{x}^{*}\right) .
\end{aligned}
$$

Таким образом, $H_{i}^{T}\left(\mathbf{x}^{*}\right) \geqslant H_{i}^{T^{0}}\left(\mathbf{x}^{*}\right)$ для всех $i$. А так как $\Gamma-$ игра с нулевой суммой, получаем равенства

$$
H_{i}^{T}\left(\mathbf{x}^{*}\right)=H_{i}^{T^{0}}\left(\mathbf{x}^{*}\right), \quad i=1, \ldots, N,
$$

и $\mathbf{x}^{*} \in N E\left(T^{0}, \Gamma\right)$. Лемма 2 доказана.

Положим для $\mathbf{x} \in X$ и для $\left(j_{1} \ldots j_{N}\right) \in S$

$$
\delta_{j_{1} \ldots j_{N}}(\mathbf{x})=T_{j_{1} \ldots j_{N}}(\mathbf{x})-T_{j_{1} \ldots j_{N}}^{0}(\mathbf{x}) .
$$

Непосредственным следствием выполнения маргинальных условий (4) являются следующее утверждение.

Лемма 3. Пусть для T выполняются маргинальные условия (4). Тогда для любой игры Г, любой ситуации $\mathbf{x} \in X$ и любого игрока $i=1, \ldots, N$ справедливо равенство

$$
H_{i}^{T}(\mathbf{x})-H_{i}^{T^{0}}(\mathbf{x})=\sum_{l=1}^{N} \sum_{j_{l}=1}^{m_{l}-1} b_{j_{1} \ldots j_{N}}^{i} \delta_{j_{1} \ldots j_{N}}(\mathbf{x}),
$$

где $b_{j_{1} \ldots j_{N}}^{i}$ - коэффициент при произведении полного набора вероятностей $x_{k j_{k}}$, $k=1, \ldots, N$, в представлении (1) функциии вылигрылиа $H_{i}^{T^{0}}$ игры $Г$.

\section{5. Основной результат}

В этом разделе устанавливается, что стохастическая независимость является единственным типом зависимости, при котором любая конечная бескоалиционная игра имеет ситуацию равновесия по Нэшу в смешанных стратегиях.

Теорема 1. Пусть $T-m_{1} \times m_{2} \times \ldots \times m_{N}$-тип зависимости. Если для любой конечной бескоалиционной игры Г размера $m_{1} \times m_{2} \times \ldots \times m_{N}$ множество $N E(T, \Gamma)$ не пусто, то $T$ представляет собой стохастическую независимость, то есть $T=T^{0}$.

Доказательство. Теорема доказывается индукцией по числу игроков. Для случая $N=2$ этот результат был установлен в работе автора [6], что послужит базой индукции.

Пусть утверждение теоремы справедливо для любого типа зависимости $N-1$ игрока. Проведем индукционный переход. Пусть $T-m_{1} \times m_{2} \times \ldots \times m_{N}$-тип зависимости, при котором $N E(T, \Gamma)$ непусто для любой игры $N$ лиц $\Gamma$. По лемме 1 также непусто множество $N E\left(T^{\mathbf{x}_{1}}, \Gamma^{1}\right)$ для любой игры $(N-1)$ лица $\Gamma^{1}$ размера $m_{2}, \ldots, m_{N}$. Отсюда по предположению индукции получаем, что для любого $k=1, \ldots, N$ введенный в предыдущем 
разделе тип зависимости $T^{\mathbf{x}_{k}}=T^{k}$ представляет собой стохастическую независимость, то есть для типа зависимости $T$ выполняются маргинальные условия (4).

Дальнейшее доказательство проводится от противного. Пусть $T \neq T^{0}$, то есть для некоторого $\left(j_{1}^{*}, \ldots, j_{N}^{*}\right) \in \prod_{i=1}^{N} S_{i}$ и некоторого $\mathbf{x}^{*} \in X=\prod_{i=1}^{N} X_{i}$ имеет место неравенство

$$
T_{j_{1}^{*} \ldots j_{N}^{*}}\left(\mathbf{x}_{1}^{*}, \ldots, \mathbf{x}_{N}^{*}\right) \neq \prod_{k=1}^{N} x_{k j_{k}^{*}}^{*}
$$

Пусть $k\left(\mathbf{x}_{i}\right)$ - число положительных компонент вектора $\mathbf{x}_{i}$ и $\operatorname{Supp} \mathbf{x}_{i}-$ множество индексов, соответствующих этим компонентам. Ясно, что $j_{i}^{*} \in \operatorname{Supp}_{i}^{*}$ для $i=1, \ldots, N$.

Не ограничивая общности, будем полагать, что

$$
\begin{array}{rlrl}
\operatorname{Supp} \mathbf{x}_{i}^{*} & =\left\{1,2, \ldots, k\left(\mathbf{x}_{i}^{*}\right)\right\}, & k\left(\mathbf{x}_{1}^{*}\right) \geqslant k\left(\mathbf{x}_{2}^{*}\right) \geqslant \ldots \geqslant k\left(\mathbf{x}_{N}^{*}\right), \\
j_{1}^{*} & =\ldots=j_{N}^{*}=1, \quad \delta_{1 \ldots 1}\left(\mathbf{x}^{*}\right)=T_{1 \ldots 1}\left(\mathbf{x}^{*}\right)-\prod_{k=1}^{N} x_{k 1}^{*}>0 .
\end{array}
$$

Случай А. Число линейно независимых компонент в спектре стратегии $\mathbf{x}_{1}^{*}$ игрока 1 не превышает суммы числа таких компонент в спектрах стратегий $\mathbf{x}_{i}^{*}$ остальных игроков,

$$
k\left(\mathbf{x}_{1}^{*}\right)-1 \leqslant \sum_{i=2}^{N}\left(k\left(\mathbf{x}_{i}^{*}\right)-1\right) .
$$

Для этого случая мы построим игру $\Gamma\left(\mathbf{x}^{*}\right)$ с нулевой суммой размера $m_{1} \times m_{2} \times \ldots \times m_{N}$, для которой $N E\left(T^{0}, \Gamma\left(\mathbf{x}^{*}\right)\right)=\left\{\mathbf{x}^{*}\right\}$ и $H_{1}^{T}\left(\mathbf{x}_{1}^{*}, \ldots, \mathbf{x}_{N}^{*}\right) \neq H_{1}^{T^{0}}\left(\mathbf{x}_{1}^{*}, \ldots, \mathbf{x}_{N}^{*}\right)$. Так как для типа зависимости $T$ выполняются маргинальные условия (4), по лемме 2 последнее неравенство влечет пустоту множества $N E\left(T, \Gamma\left(\mathbf{x}^{*}\right)\right)$, что противоречит предположению.

Построение игры $\Gamma\left(\mathbf{x}^{*}\right)$ базируется на примере игры, приведенной в работе автора [9]. Положим

$$
k_{i}=k\left(\mathbf{x}_{i}^{*}\right), \quad i=1, \ldots, N, \quad k+1=\sum_{l=2}^{N}\left(k_{l}-1\right) .
$$

Неравенство (5) приобретает вид $k_{1}-1 \leqslant k+1$. Сначала предположим, что $k_{1}>k_{3}$. Каждому набору стратегий $\mathbf{x}_{2}, \ldots, \mathbf{x}_{N}$ игроков $2, \ldots, N$ сопоставим $k$-мерный вектор $\mathbf{y}(\mathbf{x})=\left(y_{1}, y_{2}, \ldots, y_{k}\right)$,

$$
\mathbf{y}=\left(x_{22}, \ldots, x_{2 k_{2}-1}, x_{31}, \ldots, x_{3 k_{3}-1}, \ldots, x_{N 1}, \ldots, x_{N k_{N}-1}\right) \text {, }
$$

при этом игроку 2 соответствует набор индексов $\operatorname{Ind}(2)=\left\{1, \ldots, k_{2}-2\right\}$, а игрокам $i=3, \ldots, N$ соответствуют наборы индексов

$$
\operatorname{Ind}(i)=\left\{\sum_{l=2}^{i-1}\left(k_{l}-1\right), \ldots, \sum_{l=2}^{i}\left(k_{l}-1\right)-1\right\} \text {. }
$$

Усеченной стратегии $x_{22}, \ldots, x_{2 k_{2}-1}$ игрока 2 соответствует набор $y_{1}, \ldots, y_{k_{2}-2}$, а стратегии $\mathbf{x}_{i}=\left(x_{i 1}, \ldots, x_{i k_{i}-1}\right)$ игрока $i, i=3, \ldots, N$, соответствует набор $\left\{y_{j}, j \in \operatorname{Ind}(i)\right\}$.

Пусть $p-$ перестановка номеров $j=2, \ldots, k_{1}-1$ такая, что $p(j)-1$ равно остатку от деления $(k-j+2)$ на $\left(k_{1}-2\right)$, если он больше нуля, и равно $\left(k_{1}-1\right)$ в противном 
случае. Определим игру $N$ лиц $\Gamma\left(\mathbf{x}^{*}\right)=\Gamma_{c}\left(\mathbf{x}^{*}\right)$, где $c>0$, со следующими функциями выигрыша:

$$
\begin{aligned}
& H_{1}^{T^{0}}(\mathbf{x})=H_{1}(\mathbf{x})=c x_{11}\left(x_{21}-x_{21}^{*}\right) \prod_{l=3}^{N}\left(x_{l 1}+1\right)+\sum_{j=2}^{k_{1}-1} x_{1 j}\left(y_{p(j)}-y_{p(j)}^{*}\right)-2 \sum_{j=k_{1}+1}^{m_{1}} x_{1 j}, \\
& H_{2}^{T^{0}}(\mathbf{x})=H_{2}(\mathbf{x})=-c x_{21}\left(x_{11}-x_{11}^{*}\right) \prod_{l=3}^{N}\left(x_{l 1}+1\right)+\sum_{j=2}^{k_{2}-1} x_{2 j} \alpha_{j}\left(\mathbf{x}^{*}, \mathbf{x}\right)-2 \sum_{j=k_{2}+1}^{m_{2}} x_{2 j},
\end{aligned}
$$

и для игроков $i=3, \ldots, N$

$$
H_{i}^{T^{0}}(\mathbf{x})=H_{i}(\mathbf{x})=\sum_{j \in \operatorname{Ind}(i)} y_{j} \alpha_{j}\left(\mathbf{x}^{*}, \mathbf{x}\right)-2 \sum_{j=k_{i}+1}^{m_{i}} x_{i j},
$$

где $\mathbf{y}^{*}=\mathbf{y}\left(\mathbf{x}^{*}\right)$ и для $j \in \operatorname{Ind}(i)$

$$
\alpha_{j}\left(\mathbf{x}^{*}, \mathbf{x}\right)= \begin{cases}\left(x_{1 k-j+3}^{*}-x_{1 k-j+3}\right), & \text { если } j>k-k_{1}+3, \\ \left(y_{j+k_{1}-2}-y_{j+k_{1}-2}^{*}\right), & \text { если } j \leqslant k-k_{1}+3 .\end{cases}
$$

Поскольку $k_{1}>k_{3}$, справедливо неравенство $k_{1}-2>k_{l}-1$ при $l=3, \ldots, N$ и, следовательно, индексы $j$ и $\left(j+k_{1}-2\right)$ соответствуют разным игрокам: если $j \in \operatorname{Ind}(i)$, то $\left(j+k_{1}-2\right) \notin \operatorname{Ind}(i)$. Таким образом, описанные функции выигрыша задают конечную бескоалиционную игру размера $m_{1} \times m_{2} \times \ldots \times m_{N}$ в форме (1). Так как слагаемые, зависящие от стратегий всех игроков, встречаются в функциях выигрышей дважды и в сумме дают нуль, а именно, это коэффициенты при $\prod_{l=1}^{N} x_{l 1}$ в разложении (1) $b_{1 \ldots 1}^{1}=c$, $b_{1 \ldots 1}^{2}=-c$, то, не умаляя общности, игру $Г\left(\mathbf{x}^{*}\right)$ можно считать игрой с нулевой суммой.

Очевидно, что при всех $i=1, \ldots, N$ имеет место равенство

$$
\max _{\mathbf{x}_{i} \in X_{i}} H_{i}\left(\mathbf{x}^{*} \| \mathbf{x}_{i}\right)=0=H_{i}\left(\mathbf{x}^{*}\right),
$$

то есть $\mathbf{x}^{*}-$ ситуация равновесия игры $\Gamma\left(\mathbf{x}^{*}\right)$.

Поскольку для каждого игрока $i$ коэффициенты в его функции выигрыша при $x_{i j} \mathrm{c}$ номерами $j$, превышающими $k_{i}$, отрицательны, и эти коэффициенты не входят в функции выигрыша его партнеров, ввиду свойства 1 раздела 2 допущение, что $k_{i}=m_{i}$ для всех $i=1, \ldots, N$ и ситуация $\mathbf{x}^{*}$ вполне смешана, не оказывает влияние на стратегические возможности игроков.

Покажем, что ситуация равновесия $\mathbf{x}^{*}$ единственна. Пусть $\overline{\mathbf{x}}=\left(\overline{\mathbf{x}}_{1}, \ldots, \overline{\mathbf{x}}_{N}\right)-$ другая ситуация равновесия игры $\Gamma\left(\mathbf{x}^{*}\right)$. Предположим, что $\overline{\mathbf{x}}_{1} \neq \mathbf{x}_{1}^{*}$.

Вариант 1. Предположим, что для некоторого $j_{0}, 1 \leqslant j_{0} \leqslant k_{1}-1$, справедливы неравенства $\bar{x}_{1 j_{0}}>x_{1 j_{0}}^{*}>0$. Если $j_{0}=1$, то есть $\bar{x}_{11}>x_{11}^{*}>0$, по свойству 1 раздела 2 $\bar{x}_{21}=0$, следовательно, $\bar{x}_{11}=0$, что невозможно.

Пусть $2 \leqslant j_{0} \leqslant k_{1}-1$. Положим $j_{1}=k-j_{0}+3$. Для некоторого $i_{1}, 2 \leqslant i_{1} \leqslant N$, справедливо включение $j_{1} \in \operatorname{Ind}\left(i_{1}\right)$.

Рассмотрим функцию выигрыша игрока $i_{1}$ при фиксированных стратегиях $\overline{\mathbf{x}}_{i}$ других игроков $H_{i_{1}}\left(\overline{\mathbf{x}} \| \mathbf{x}_{i_{1}}\right)$. Так как $j_{1}>k-k_{1}+3$, получаем, что, коэффициент при $y_{j_{1}}$, равный $x_{1 k-j_{1}+3}^{*}-\bar{x}_{11 k-j_{1}+3}=x_{1 j_{0}}^{*}-\bar{x}_{1 j_{0}}$, отрицателен. Отсюда по свойству 1 раздела 2 следует, что $\bar{y}_{j_{1}}=0$, где $\overline{\mathbf{y}}=\mathbf{y}(\overline{\mathbf{x}})$. 
Если $j_{1}>k_{1}-1$, то положим $j_{2}=j_{1}-\left(k_{1}-2\right)$. Для некоторого $i_{2}, 2 \leqslant i_{2} \leqslant N$, справедливо включение $j_{2} \in \operatorname{Ind}\left(i_{2}\right)$. Далее, рассмотрев $H_{i_{2}}\left(\overline{\mathbf{x}} \| x_{i_{2}}\right)$, находим, что $\bar{y}_{j_{2}}=0$.

Если $j_{2}>k_{1}-1$, то продолжим этот процесс дальше, положив $j_{3}=j_{2}-\left(k_{1}-2\right)$. Через конечное число шагов мы придем к случаю $\bar{y}_{j_{n}}=0, j_{n} \leqslant k_{1}-1$. Легко видеть, что в соответствии с определением $p(j)$

$$
k_{1}-1 \geqslant j_{n}=j_{1}-(n-1)\left(k_{1}-2\right)=1+\left(k-j_{0}+2\right)-(n-1)\left(k_{1}-2\right)=p\left(j_{0}\right),
$$

то есть $\bar{y}_{p\left(j_{0}\right)}=0$.

Рассмотрим теперь функцию выигрыша игрока 1 при фиксированных стратегиях $\overline{\mathbf{x}}_{i}$ других игроков. Так как коэффициент при $x_{1 j_{0}}$, равный $\bar{y}_{p\left(j_{0}\right)}-y_{p\left(j_{0}\right)}^{*}=-y_{p\left(j_{0}\right)}^{*}$, отрицателен, как и прежде, получаем $\bar{x}_{1 j_{0}}=0$, что противоречит предположению $\bar{x}_{1 j_{0}}>x_{1 j_{0}}^{*}>0$.

Вариант 2. Для всех $j, 1 \leqslant j \leqslant k_{1}-1$, выполняются неравенства $\bar{x}_{1 j} \leqslant x_{1 j}^{*}$, и следовательно, $\bar{x}_{1 k_{1}} \geqslant x_{1 k_{1}}^{*}>0$. Принимая во внимание свойство 2 раздела 2, получаем, что коэффициенты при $x_{1 j}$ в функции выигрыша $H_{1}\left(\overline{\mathbf{x}} \| \mathbf{x}_{1}\right)$ не положительны, то есть $\bar{x}_{21}-x_{21}^{*} \leqslant 0$ и при всех $j=2, \ldots, k_{1}-1$ выполняются неравенства $\bar{y}_{p(j)}-y_{p(j)}^{*} \leqslant 0$ или, что то же самое,

$$
\bar{x}_{2 j}-x_{2 j}^{*} \leqslant 0, \quad 1 \leqslant j \leqslant k_{1}-1 .
$$

Далее, поскольку $\overline{\mathbf{x}}_{1} \neq \mathbf{x}_{1}^{*}$, существует $j_{0}, 1 \leqslant j_{0} \leqslant k_{1}-1$, для которого $\bar{x}_{1 j_{0}}<x_{1 j_{0}}^{*}$. Отсюда, как и в варианте 1 , получим, что $\bar{x}_{i k_{i}}=0$ при некотором $i=2, \ldots, N$. Так как $x_{i k_{i}}^{*}>0$, то либо $\bar{x}_{21}>x_{21}^{*}$, что противоречит (6), либо для некоторого $j, j \in \operatorname{Ind}(i)$, имеет место неравенство $\bar{y}_{j}>y_{j}^{*}$.

Если $j \leqslant k_{1}-1$, то также получаем противоречие с (6). В противном случае $i \geqslant 3$ и, положив $j_{1}=j-\left(k_{1}-2\right)$, снова получим, что $\bar{x}_{i_{1} k_{i_{1}}}=0$, где $i_{1}$ определяется условием $j_{1} \in \operatorname{Ind}\left(i_{1}\right)$. Следовательно, существует $j_{1}^{\prime} \in \operatorname{Ind}\left(i_{1}\right)$, для которого $\bar{y}_{j_{1}^{\prime}}>y_{j_{1}^{\prime}}^{*}$.

Заметим, что $i_{1}<i$, поскольку $k_{1}-2>k_{l}-1$ при $l=3, \ldots, N$ и, следовательно, $j_{1}^{\prime}<j$. Продолжая этот процесс, для некоторого конечного $n$ мы получим $j_{n}^{\prime} \leqslant k_{1}-1$ и $\bar{y}_{j_{n}^{\prime}}>y_{j_{n}^{\prime}}^{*}$, что противоречит (6).

Разбор вариантов 1 и 2 при $k_{1}>k_{3}$ показал, что $\overline{\mathbf{x}}_{1}=\mathbf{x}_{1}^{*}$. Следовательно, по свойству 3 раздела 2 получаем, что $\bar{x}_{21}=x_{21}^{*}$ и для всех $j=1, \ldots, k_{1}-2$, выполняются равенства $\bar{y}_{j}=y_{j}^{*}$.

Если $k_{1}-1=k+1$, то это уже означает совпадение ситуаций равновесия $\overline{\mathbf{x}}$ и $\mathbf{x}^{*}$.

Пусть $k_{1}-1<k+1$. Так как $k_{2} \leqslant k_{1}$, справедливо равенство $\bar{x}_{2 j}=x_{2 j}^{*}$ при всех $1 \leqslant j \leqslant k_{2}$, то есть стратегии $\overline{\mathbf{x}}_{2}$ и $\mathbf{x}_{2}^{*}$ игрока 2 совпадают и, следовательно, стратегия $\overline{\mathbf{x}}_{2}$ игрока 2 вполне смешана. Снова по свойству 3 получаем, что $\bar{y}_{j+k_{1}-2}-y_{j+k_{1}-2}^{*}=0$ для всех $j, 1 \leqslant j \leqslant \min \left(k_{2}-1, k-k_{1}+3\right)$. Если $k-\left(k_{1}-2\right) \leqslant k_{2}-2$, то это означает, что $\bar{y}_{j}=y_{j}^{*}$ для всех $j=1, \ldots, k$, то есть ситуации равновесия $\overline{\mathbf{x}}$ и $\mathbf{x}^{*}$ совпадают. Если же $k-\left(k_{1}-2\right)>k_{2}-2$, то, так как $k_{3} \leqslant k_{2}$, справедливо равенство $\bar{x}_{3 j}=x_{3 j}^{*}$ при всех $1 \leqslant j \leqslant k_{3}$, то есть стратегии $\overline{\mathbf{x}}_{3}$ и $\mathbf{x}_{3}^{*}$ игрока 3 совпадают. Аналогично получаем, что $\overline{\mathbf{x}}_{4}=\mathbf{x}_{4}^{*}, \ldots, \overline{\mathbf{x}}_{N}=\mathbf{x}_{N}^{*}$. Таким образом, ситуация равновесия $\overline{\mathbf{x}}$ совпадает с ситуацией равновесия $\mathbf{x}^{*}$.

Для завершения рассмотрения случая А предположим, что $k_{1}=k_{2}=k_{3}=\ldots=$ $k_{l}>k_{l+1} \geqslant \ldots \geqslant k_{N}$ при некотором $3 \leqslant l \leqslant N$. Тогда перепишем $k$-мерный вектор $\mathbf{y}(\mathbf{x})=\left(y_{1}, y_{2}, \ldots, y_{k}\right)$, сопоставляемый набору стратегий $\mathbf{x}_{2}, \ldots, \mathbf{x}_{N}$ игроков $2, \ldots, N$, следующим образом, перенеся компоненты $x_{31}, \ldots, x_{l 1}$ на последние места:

$$
\mathbf{y}=\left(x_{22}, \ldots, x_{2 k_{2}-1}, x_{32}, \ldots, x_{3 k_{3}-1}, \ldots, x_{N k_{N}-1}, x_{31}, \ldots, x_{l 1}\right) \text {. }
$$


При такой перестановке индексы $j$ и $j+k_{1}-2$ в определении функций выигрышей игры $\Gamma\left(\mathbf{x}^{*}\right)$ по-прежнему соответствуют разным игрокам: если $j \in \operatorname{Ind}(i)$, то $j+k_{1}-2 \notin \operatorname{Ind}(i)$. Эти функции выигрыша задают конечную бескоалиционную игру размера $m_{1} \times m_{2} \times \ldots \times m_{N}$ в форме (1) с единственной ситуацией равновесия $\mathbf{x}^{*}$ при стохастической независимости выборов игроков $T^{0}$. С помощью рассуждений, аналогичных вышеизложенным, получим, что ситуация $\mathbf{x}^{*}$ не является равновесной при типе зависимости $T$. Следовательно, множество $N E\left(T, \Gamma\left(\mathbf{x}^{*}\right)\right)$ пусто, что противоречит предположению.

Таким образом, мы установили, что если для любой игры $\Gamma$ множество $N E(T, \Gamma)$ непусто, то в ситуациях $\mathbf{x}$, для которых выполнено условие (5), справедливо равенство $T(\mathbf{x})=T^{0}(\mathbf{x})$.

Случай Б. Для стратегии $\mathbf{x}^{*}$, в которой по предположению $T\left(\mathbf{x}^{*}\right) \neq T^{0}\left(\mathbf{x}^{*}\right)$, не выполняется соотношение (5), то есть

$$
k\left(\mathbf{x}_{1}^{*}\right)-1=k_{1}-1>\sum_{i=2}^{N}\left(k\left(\mathbf{x}_{i}^{*}\right)-1\right)=k+1 .
$$

В этом случае, вообще говоря, игра $\Gamma\left(\mathbf{x}^{*}\right)$ такова, что $N E\left(T^{0}, \Gamma\left(\mathbf{x}^{*}\right)\right)=\left\{\mathbf{x}^{*}\right\}$ не существует. Действительно, при $N=2$ (биматричные игры) неравенство (7) имеет вид

$$
k_{1}=k\left(\mathbf{x}_{1}^{*}\right)>k_{2}=k\left(\mathbf{x}_{2}^{*}\right) .
$$

В работе автора [10] установлено, что равенство $k_{1}=k_{2}$ является необходимым и достаточным условием существования биматричной игры с единственной ситуацией равновесия $\mathbf{x}^{*}$.

Рассмотрим стратегию игрока 1

$$
\mathbf{x}_{1}^{* *}=\left(x_{11}^{*}, \ldots, x_{1 k+1}^{*}, \sum_{l=k+2}^{k_{1}} x_{1 l}^{*}, 0, \ldots, 0\right) .
$$

Положим $\mathbf{x}^{* *}=\left(\mathbf{x}_{1}^{* *}, \mathbf{x}_{2}^{*}, \ldots, \mathbf{x}_{N}^{*}\right)$. Ситуация $\mathbf{x}^{* *}$ соответствует случаю А,

$$
k\left(\mathbf{x}_{1}^{* *}\right)-1=\sum_{i=2}^{N}\left(k\left(\mathbf{x}_{i}^{*}\right)-1\right)=k+1 .
$$

Согласно случаю А, при всех $j_{1}, \ldots, j_{N}$

$$
T_{j_{1} \ldots j_{N}}\left(\mathbf{x}^{* *}\right)=T_{j_{1} \ldots j_{N}}\left(\mathbf{x}^{* *}\right) .
$$

Положим

$$
c=4 \frac{\sum_{j=k+3}^{k_{1}} x_{1 j}^{*}}{\delta_{1 \ldots 1}\left(\mathbf{x}^{*}\right)} .
$$

Параметр $c$ положителен, так как $k\left(\mathbf{x}_{1}^{*}\right)=k_{1} \geqslant k+3$ и

$$
\delta_{1 \ldots 1}\left(\mathbf{x}^{*}\right)=T_{1 \ldots 1}\left(\mathbf{x}^{*}\right)-T_{1 \ldots 1}^{0}\left(\mathbf{x}^{*}\right)>0 .
$$

Рассмотрим игру $\Gamma_{c}\left(\mathbf{x}^{* *}\right)$ (см. случай А). Справедливы соотношения

$$
N E\left(T, \Gamma_{c}\left(\mathbf{x}^{* *}\right)\right) \subset N E\left(T^{0}, \Gamma_{c}\left(\mathbf{x}^{* *}\right)\right)=\left\{\mathbf{x}^{* *}\right\} .
$$


Рассмотрим для этой игры функцию выигрыша игрока 1

$$
H_{1}^{T}\left(\mathbf{x}^{* *}\right)=H_{1}^{T^{0}}\left(\mathbf{x}^{* *}\right)=0, \quad H_{1}\left(\mathbf{x}^{*}\right)=-2 \sum_{j=k+3}^{k_{1}} x_{1 j}^{*},
$$

и покажем, что

$$
H_{1}^{T}\left(\mathbf{x}^{* *}\right)<H_{1}^{T}\left(\mathbf{x}^{* *} \| \mathbf{x}_{1}^{*}\right)=H_{1}^{T}\left(\mathbf{x}^{*}\right) .
$$

Действительно,

$$
H_{1}^{T}\left(\mathbf{x}^{* *}\right)-H_{1}^{T}\left(\mathbf{x}^{*}\right)=\left(H_{1}^{T^{0}}\left(\mathbf{x}^{* *}\right)-H_{1}^{T^{0}}\left(\mathbf{x}^{*}\right)\right)-\left(H_{1}^{T}\left(\mathbf{x}^{*}\right)-H_{1}^{T^{0}}\left(\mathbf{x}^{*}\right)\right),
$$

и, используя лемму 3 , получаем, что эта разность равна

$$
2 \sum_{j=k+3}^{k_{1}} x_{1 j}^{*}-c \delta_{1 \ldots 1}\left(\mathbf{x}^{*}\right)=-2 \sum_{j=k+3}^{k_{1}} x_{1 j}^{*}<0 .
$$

Следовательно, множество $N E\left(T, \Gamma_{c}\left(\mathbf{x}^{* *}\right)\right)$ пусто.

Полученное противоречие завершает доказательство теоремы.

\section{Список литературы}

1. von Neumann J., Zur Theorie der Gesellschaftspiele. Math. Ann. (1928) 100, 295-320.

2. Nash J., Non-cooperative games. Ann. Math., II Ser. (1951) 54, 286-295.

3. Нэш Дж., Бескоалиционные игры. В сб.: Матричные игры. Физматгиз, Москва, 1961, с. 205-221.

4. Кац М., Статистическая независимость в теории вероятностей, анализе и теории чисел. ИЛ, Москва, 1963.

5. Воробьев Н. Н., Аналитическая характеризация независимости и марковости. Теория вероятностей и ее применения (1961) 6, №4, 422-426.

6. Kreps V., On games with stochastically dependent strategies. Int. J. Game Theory (1994) 23, 57-64.

7. Воробьев Н. Н., Основы теории игр. Бескоалиционные игры. Наука, Москва, 1984.

8. Печерский С. Л., Беляева А. А., Теория игр для экономистов. Вводный курс. Изд-во Европейского университета, Санкт-Петербург, 2001.

9. Kreps V., Finite $N$-person non-cooperative games with unique equilibrium points. Int. J. Game Theory (1981) 10, №3/4, 125-129.

10. Kreps V., Bimatrix games with unique equilibrium points. Int. J. Game Theory (1974) 3, №2, $115-118$. 